\title{
Excitations from a chiral magnetized state of a frustrated quantum spin liquid
}

\author{
A. Zheludev, ${ }^{1, *}$ V. O. Garlea, ${ }^{1}$ A. Tsvelik, ${ }^{2}$ L.-P. Regnault, ${ }^{3}$ K. Habicht, ${ }^{4}$ K. Kiefer, ${ }^{4}$ and B. Roessli ${ }^{5}$ \\ ${ }^{1}$ Neutron Scattering Sciences Division, Oak Ridge National Laboratory, Oak Ridge, Tennessee 37831, USA \\ ${ }^{2}$ Department of Condensed Matter Physics and Materials Science, Brookhaven National Laboratory, Upton, New York 11973, USA \\ ${ }^{3}$ INAC-SPSMS-MDN, CEA-Grenoble, 17 rue des Martyrs, 38054 Grenoble Cedex 9, France \\ ${ }^{4}$ Helmholtz-Zentrum Berlin für Materialien und Energie, D-14109 Berlin, Germany \\ ${ }^{5}$ Laboratory for Neutron Scattering, ETH Zürich and Paul Scherrer Institut, Villigen, Switzerland
}

(Received 18 September 2009; published 15 December 2009)

\begin{abstract}
We study excitations in weakly interacting pairs of quantum spin ladders coupled through geometrically frustrated bonds. The ground state is a disordered spin liquid that at high fields is replaced by an ordered chiral helimagnetic phase. The spectra observed by high-field inelastic neutron scattering experiments on the prototype compound $\mathrm{Sul}-\mathrm{Cu}_{2} \mathrm{Cl}_{4}$ are qualitatively different from those in the previously studied frustration-free spin liquids. Beyond the critical field $H_{c}=3.7 \mathrm{~T}$, the soft mode that drives the quantum phase transition spawns two separate excitations: a gapless Goldstone mode and a massive magnon. Additional massive quasiparticles are clearly visible below $H_{c}$, but are destroyed in the ordered phase. In their place one observes a sharply bound excitation continuum.
\end{abstract}

DOI: 10.1103/PhysRevB.80.214413

PACS number(s): 75.10.Jm, 75.40.Gb, 73.43.Nq, 78.70.Nx

\section{INTRODUCTION}

Gapped quantum-disordered antiferromagnets (AFs), also known as "spin liquids," have become prototype materials for the study of Bose-Einstein condensation and related phenomena. ${ }^{1-6}$ By virtue of the Zeeman effect, the magnetic field directly affects the chemical potential for the relevant lowest-energy triplet excitations. The latter excitations can be viewed as bosons with hard-core repulsion. Exotic quantum critical points are realized when the gap energy for one member of the triplet is driven to zero at some critical field $H_{c}$ and a macroscopic number of those magnons get incorporated into the ground state. At $H>H_{c}$ one typically observes a magnetically ordered state. However, due to the strong interactions the corresponding excitation spectrum is nothing like that of conventional ordered AFs. ${ }^{6}$

Usually at $H>H_{c}$ the magnon branch that goes soft at the quantum critical point is replaced by a gapless collective Goldstone mode ${ }^{4-6}$ For all models and prototypical materials studied to date, the two other members of the original excitation triplet survive as sharp gap excitations. Thus, the low-energy spectrum remains dominated by three long-lived quasiparticles. In the present work we study the spin dynamics in a gapped quantum $\mathrm{AF}$ with strong geometric frustration of magnetic interactions. In a stark contrast to the behavior of frustration-free spin liquids, we find that above $H_{c}$ the soft mode gives rise to two distinct excitation branches, while the higher-energy gapped magnons become unstable and are replaced by a broad excitation continuum.

Our prototype material, $\mathrm{Sul}-\mathrm{Cu}_{2} \mathrm{Cl}_{4}{ }^{7-9}$ is a quasi-onedimensional Heisenberg AF with a singlet ground state and a spin gap of $\Delta_{0}=0.52 \mathrm{meV}$. The corresponding network of $S=1 / 2 \mathrm{Cu}^{2+}$ spins is best described as an array of four-leg spin tubes with dominant $\mathrm{AF}$ nearest-neighbor coupling along the legs and several weaker rung interactions of comparable strength. The tube's legs run along the crystallographic $c$ axis of the triclinic $P \overline{1}$ structure. The corresponding interaction topology is illustrated in Fig. 1. Each spin tube consists of two spin ladders, $J_{1}$ and $J_{3}$ being the leg and the rung exchange constants, respectively. The two ladders are coupled via exchange constants $J_{2}$ and $J_{4}$, that are in obvious geometric frustration with $J_{1}$. Even though the ground state is disordered, frustration ensures that dynamic one-dimensional spin correlations in Sul- $\mathrm{Cu}_{2} \mathrm{Cl}_{4}$ are peaked at incommensurate wave vectors $l_{0}=0.5-\delta, 1.5+\delta, \delta=0.022(2){ }^{8}$ The dispersion of the gap excitation along the legs of the tubes can be approximated as

$$
\left(\hbar \omega_{\mathbf{q}}\right)^{2}=\Delta_{0}^{2}+v_{0}^{2}\left(\mathbf{q c}-2 \pi l_{0}\right)^{2},
$$

where $v_{0} \sim 14 \mathrm{meV}$ is the spin wave velocity. Any dispersion transverse to the $c$ axis is undetectably small. Previously we have demonstrated that the application of a magnetic field exceeding $H_{c}=3.7 \mathrm{~T}$ destroys the spin liquid and induce an ordered helimagnetic state with an incommensurate propagation vector. ${ }^{9}$ The incommensurability along the $c$ axis exactly corresponds to the minimum of the dispersion at zero field. The present work deals with the spin dynamics on either side of this field-induced phase transition.

\section{EXPERIMENTAL PROCEDURES}

Inelastic neutron scattering measurements were performed on the same fully deuterated single crystal samples as those used in Ref. 8. Two series of experiments were carried out at the V2-Flex and TASP cold neutron spectrometers at HMI and PSI, respectively. In setup 1 we used

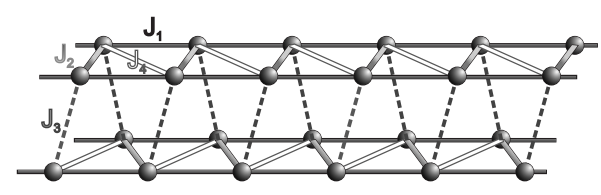

FIG. 1. A schematic representation of the magnetic interaction topology in $\mathrm{Sul}-\mathrm{Cu}_{2} \mathrm{Cl}_{4} . J_{1}$ denotes the "leg" coupling, and $J_{2}, J_{3}$ and $J_{4}$ are the three distinct "rung" interactions. 
$E_{f}=3.7 \mathrm{meV}$ fixed final energy neutrons, while the sample environment was a $14.5 \mathrm{~T}$ split-coil cryomagnet with a dilution refrigerator insert. Data in setup 2 were collected with $E_{f}=3 \mathrm{meV}$ and a similar $9 \mathrm{~T}$ setup. In both cases we utilized pyrolitic graphite (PG) vertically focused monochromators and horizontally focused analyzers, as well as Be filters positioned after the sample. The field was in all cases applied along the $b$ axis and scattering data were collected in the $(h, 0, l)$ reciprocal-space plane. Most of the data were measured in constant $q$ scans at the wave vector transfers where the previously measured one-dimensional (1D) magnon dispersion is a minimum: $q=(h, 0,0.48)$. Momentum transfers along the $a^{*}$ axis were chosen to optimize wave-vector resolution along the $c^{*}$ direction. The background was measured at the wave vectors $(h, 0,0.6)$, where no magnetic contribution is expected, due to a very steep dispersion along the crystallographic $c^{*}$ direction. These background scans were fit to a constant plus a Gaussian function centered at zero energy transfer, to account for fast neutron background and elastic incoherent scattering from the sample and sample environment, respectively. The resulting fitted background function was subtracted from the signal scans.

\section{EXPERIMENTAL RESULTS}

Typical background-subtracted scans collected at $H<H_{c}$ are shown in Fig. 2. As the external magnetic field is increased, the single peak seen at $H=0$ (Ref. 8, Fig. 3) splits into three components. The gap energy of the central and most intense component (mode 2) is field independent. The energies of modes 1(3) decrease (increase) with the field, as expected for Zeeman splitting of the $S=1$ excitation triplet. The gap in the lower branch (mode 1) approaches zero energy as $H \rightarrow H_{c}$.

For magnetic fields exceeding $H_{c}=3.7 \mathrm{~T}$ (Fig. 3) the measured excitation spectrum undergoes some drastic changes. As can be seen in Fig. 3(a), just above the critical field, at $H=4 \mathrm{~T}$, mode 3 becomes very weak and is, in fact, barely visible. It is totally absent for all other values of magnetic field applied in our experiments $(5,8,9.5$, and $13.5 \mathrm{~T})$. The gap energy for mode 2 starts to increase with increasing $H$. Finally, mode 1 , being critical at $H=H_{c}$, re-acquires an energy gap at higher fields [Figs. 3(b) and 3(c)]. This gap, however, increases rather slowly with the field not exceeding $0.3 \mathrm{meV}$ even at the highest attainable field of $13.5 \mathrm{~T}$.

\section{THEORETICAL CONSIDERATIONS}

The reopening of the spin gap at $H>H_{c}$ is, at the first glance, puzzling. Indeed, in those spin liquids that undergo similar field-induced ordering transitions the gap reappears in the high field phase due to magnetic anisotropy effects. In that case the transition is of the Ising, rather than the BEC, universality class. However, in the case of $\mathrm{Sul}_{-} \mathrm{Cu}_{2} \mathrm{Cl}_{4}$, magnetic anisotropy is negligibly small, as discussed in Ref. 9. Moreover, since the helimagnetic ordering occurs at an incommensurate wave vector, one can rigorously prove that there must be a gapless "sliding mode" ("phason"). This remains true even if anisotropy is present. The sinusoidal spin
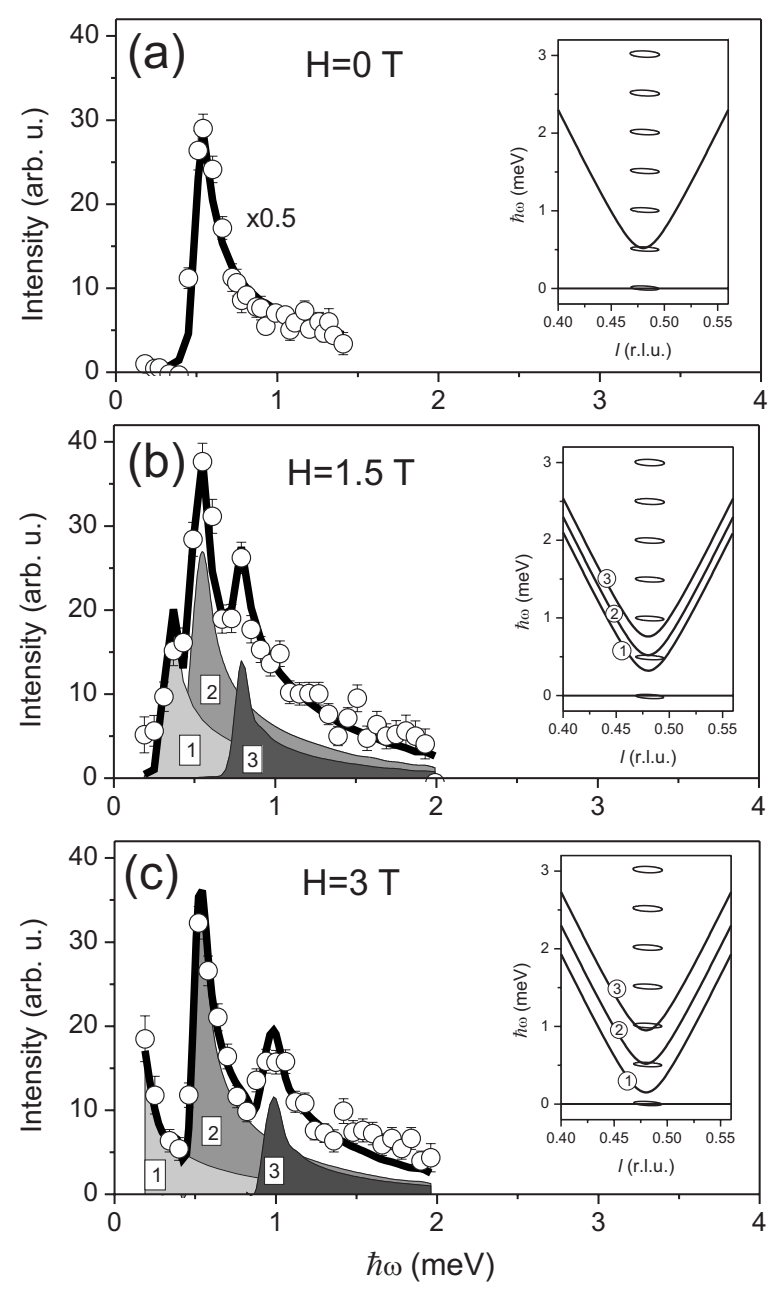

FIG. 2. Field dependence of background-subtracted inelastic scattering at the $1 \mathrm{D} \mathrm{AF}$ zone-center $(0.5,0,0.48)$, collected at $H<H_{c}$ and $\mathrm{T} \approx 70 \mathrm{mK}$. Solid lines are model cross section fits, as described in Sec. V. Shaded areas are partial contributions of three separate excitation branches. Inserts: Dispersions of the three fielddriven excitation branches in Sul- $\mathrm{Cu}_{2} \mathrm{Cl}_{4}$ and evolution of the calculated full width at half maximum resolution ellipsoids plotted in projection onto the $(l, \hbar \omega)$ plane.

spiral that is the ground state in the isotropic case, will be distorted by the anisotropy to form a soliton lattice. That soliton array, however, being incommensurate, will retain the ability to slide freely relative to the underlying crystal structure.

To fully understand the nature of the spin gap in Sul- $\mathrm{Cu}_{2} \mathrm{Cl}_{4}$, we shall develop a field-theoretical description of the low-energy spin correlations in a pair of magnetized spin ladders coupled with geometrically frustrated interactions.

\section{A. Interladder interactions}

We start with writing down the spin Hamiltonian for the frustrated spin tube geometry shown in Fig. 1

$$
\begin{aligned}
H= & \sum_{n ; a=1,2}\left[J_{1} \mathbf{S}_{n}^{a} \mathbf{S}_{n+1}^{a}+J_{1} \mathbf{T}_{n}^{a} \mathbf{T}_{n+1}^{a}+J_{3} \mathbf{S}_{n}^{a} \mathbf{T}_{n}^{a}\right] \\
& +\sum_{n}\left[J_{4} \mathbf{S}_{n}^{1} \mathbf{S}_{n}^{2}+J_{2} \mathbf{S}_{n+1}^{1} \mathbf{S}_{n}^{2}+J_{2} \mathbf{T}_{n}^{1} \mathbf{T}_{n}^{2}+J_{4} \mathbf{T}_{n+1}^{1} \mathbf{T}_{n}^{2}\right] .
\end{aligned}
$$



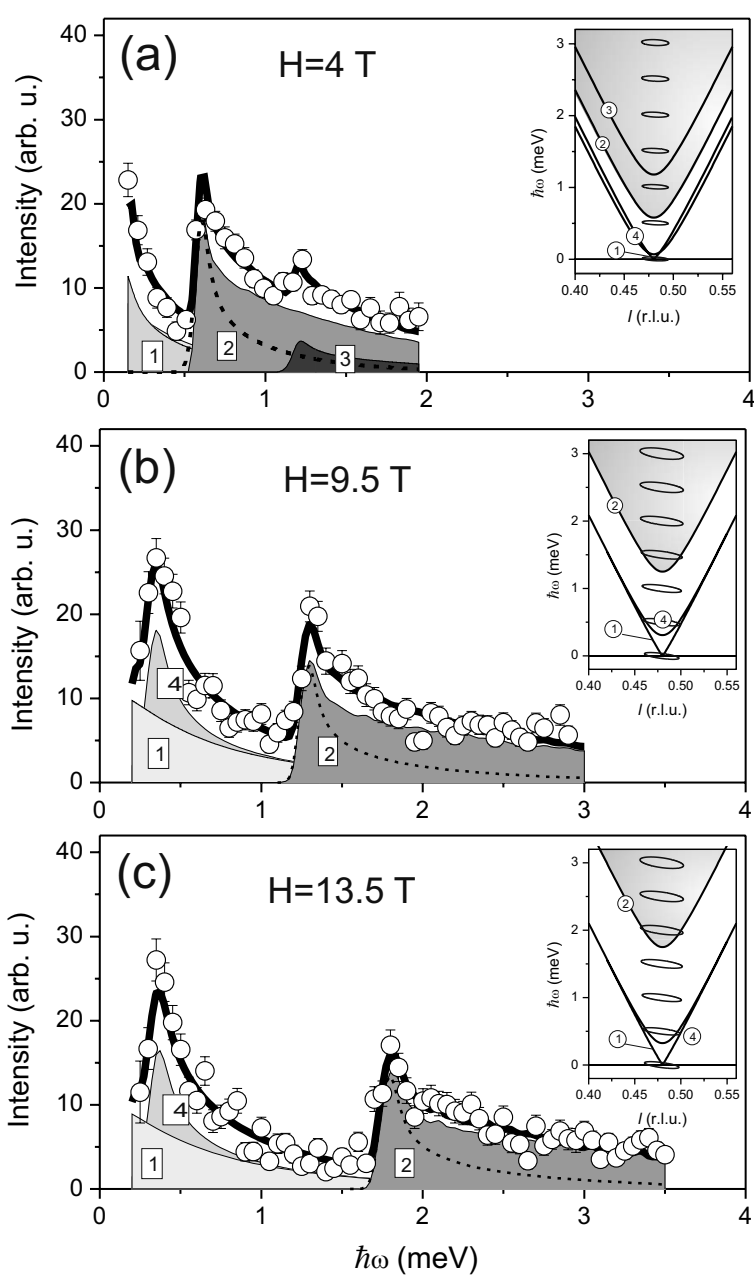

FIG. 3. Background-subtracted neutron spectra measured in $\mathrm{Sul}-\mathrm{Cu}_{2} \mathrm{Cl}_{4}$ at the $1 \mathrm{D} \mathrm{AF}$ zone center $(0.5,0,0.48)$ in various applied magnetic fields above the critical field, $H>H_{c}$, and $150 \mathrm{mK}$. The lines, shaded areas, and graphical representations of the resolution functions are as in Fig. 2. The shaded area in the inserts indicates a continuum scattering, with the corresponding solid line depicting the lower bound of the continua.

Here $a=1,2$ labels the two structural ladders, while $\mathbf{S}_{n}$ an $\mathbf{T}_{n}$ are spin operators on the corresponding legs. We direct the reader's attention to the fact that the ratio of spin gap $\Delta_{0}$ to the magnetic excitations bandwidth $v_{0}$ in $\mathrm{Sul}-\mathrm{Cu}_{2} \mathrm{Cl}_{4}$ is much smaller than in most known spin ladders. This is likely to be a consequence of geometric frustration. Whatever the cause, it makes this particular system ideal for application of the field theory methods developed in Ref. 10.

We shall treat Hamiltonian (2) as one describing two weakly interacting ladders. Assuming $J_{1} \gg J_{2}, J_{3}, J_{4}$, there are two cases to consider: (i) $J_{3} \gg\left|J_{2}-J_{4}\right| \equiv J_{\perp}$. Here one ladder is composed of spins $\mathbf{S}_{1,2}$ and the other of spins $\mathbf{T}_{1,2}$. Such ladders have spectral gaps $\Delta \sim J_{3}$. The second sum in Eq. (2) represents a frustrated interaction and in the gapped phase can be considered as a perturbation. This is justified by the fact that the term with $J_{3}$ has smaller scaling dimension than $J_{2}, J_{4}$ hence generating a stronger coupling. (ii) Alternatively, one may consider $\left|J_{2}-J_{4}\right| \gg J_{3} \equiv J_{\perp}$. Then the two ladders are composed of $\mathbf{S}$ and $\mathbf{T}$ spins, respectively. The spin gap is of the order of $\left|J_{2}-J_{4}\right|$. In either scenario, the interladder interactions $J_{\perp}$ are irrelevant at small magnetic fields, yet become relevant when the external field drives the single-ladder gap to zero.

To obtain the continuum limit of the lattice model (2), we approximate the spin operators as follows:

$$
\begin{aligned}
\mathbf{S}_{n}^{a} & \approx \mathbf{M}^{a}(x)+(-1)^{n} \mathbf{n}^{a}(x), \quad a=1,2, \\
\mathbf{T}_{n}^{a} \approx \mathbf{L}^{a}(x)+(-1)^{n} \mathbf{l}^{a}(x), & a=1,2,
\end{aligned}
$$

where $\mathbf{I}, \mathbf{n}$ and $\mathbf{L}, \mathbf{M}$ are operators with scaling dimensions $1 / 2$ and 1 , respectively. It turns out that only antibonding combinations of the staggered magnetizations become critical in high magnetic fields. Therefore only such combinations will generate relevant inter-ladder coupling at $H>H_{c}$. They are defined as $\mathbf{N}_{a}=\mathbf{n}^{a}-\mathbf{l}^{a}(a=1,2)$ and $\mathbf{N}_{1}=\mathbf{n}_{1}-\mathbf{n}_{2}, \mathbf{N}_{2}=\mathbf{l}_{1}-\mathbf{l}_{2}$, for cases (i) and (ii), respectively.

Substituting these definitions into Eq. (2) and keeping only the antibonding staggered magnetization terms, we obtain the following interladder interaction:

$$
V=\gamma \int \mathrm{d} x\left[\mathbf{N}_{1} \partial_{x} \mathbf{N}_{2}-\mathbf{N}_{2} \partial_{x} \mathbf{N}_{1}\right],
$$

where $\gamma \sim J_{\perp} / J_{1}$.

\section{B. Ginzburg-Landau model}

Interaction (4) is easier to treat in the spin-liquid phase when the spectrum is gapped. Here we shall follow Affleck ${ }^{11}$ who suggested to describe the low lying modes of spin ladder by an effective Ginzburg-Landau model with the Lagrangian density

$$
\mathcal{L}=\frac{1}{2}\left(\partial_{\tau} \boldsymbol{\Phi}\right)^{2}+\frac{\widetilde{v}^{2}}{2}\left(\partial_{x} \boldsymbol{\Phi}\right)^{2}+\frac{\widetilde{\Delta}^{2}}{2} \boldsymbol{\Phi}^{2}+g\left(\boldsymbol{\Phi}^{2}\right)^{2},
$$

where $\boldsymbol{\Phi} \sim \mathbf{N}$, and the bare parameters $\widetilde{v}, \widetilde{\Delta}$ are of the same order as the velocity and gap in the actual single-ladder spectrum. It is straightforward to show that the introduction of the interaction (4) generates two modes with the spectrum

$$
E_{ \pm}^{2}=\Delta^{2}+v^{2}(\mathbf{q c}-\pi \pm \delta)^{2},
$$

where $\Delta, v$ are the renormalized gap energy and spin wave velocity, respectively, and $\delta \sim \gamma$. The incommensurate gapped dispersion is fully in agreement with the existing data on $\mathrm{Sul}-\mathrm{Cu}_{2} \mathrm{Cl}_{4}$.

When the magnetic field exceeds the spin gap the transverse components of the staggered magnetizations become critical. Interaction (4) becomes relevant, but its form is highly nonstandard from the field theory point of view since the corresponding interaction density has nonzero conformal spin (that is its left and right conformal dimensions do not coincide). Attempts to treat such interactions using standard field theory methods have met with mixed success. ${ }^{12}$ Below we shall adopt a different approach. Again, following Affleck, ${ }^{11}$ close to the critical field we will describe each spin ladder using the Landau-Ginzburg theory with the $|\phi|^{4}$ interaction. When applying this formalism to our case, one re- 
places $N^{z}=\left(1-\psi^{+} \psi\right), N^{ \pm}=\left(\psi, \psi^{+}\right)$, where $\psi$ are bosonic fields and writes the Lagrangian as

$$
\begin{aligned}
\mathcal{L}= & \psi_{a}^{+}\left(\partial_{\tau}-\mu\right) \psi_{a}+\frac{1}{2 m} \partial_{x} \psi_{a}^{+} \partial_{x} \psi_{a}+g\left(\left|\psi_{1}\right|^{4}+\left|\psi_{2}\right|^{4}\right) \\
& +J_{\perp}\left(\psi_{1}^{+} \partial_{x} \psi_{2}-\psi_{2}^{+} \partial_{x} \psi_{1}\right),
\end{aligned}
$$

where $\mu \sim H-H_{c}, m \sim \Delta$, and the coupling $g$ is assumed to be large.

\section{Incommensurate order and spectrum at $\boldsymbol{H}>\boldsymbol{H}_{c}$}

We now do the substitution

$$
\psi=\frac{1}{\sqrt{2}}\left(I+\mathrm{i} \sigma_{x}\right) \tilde{\psi},
$$

and

$$
\tilde{\psi}_{1,2}=\mathrm{e}^{ \pm \mathrm{i} Q x} \eta_{1,2},
$$

where $\sigma^{x}$ is the Pauli matrix and $Q \sim \gamma$. Then the interaction density becomes

$$
\begin{aligned}
g\left(\left|\psi_{1}\right|^{4}+\left|\psi_{2}\right|^{4}\right) \rightarrow \frac{g}{2}\left[\left(\left|\eta_{1}\right|^{2}+\left|\eta_{2}\right|^{2}\right)^{2}\right. \\
\left.-\left(\eta_{1}^{+} \eta_{2} \mathrm{e}^{2 \mathrm{i} Q x}-\eta_{1}^{+} \eta_{2} \mathrm{e}^{-2 \mathrm{i} Q x}\right)^{2}\right] \\
\rightarrow \frac{g}{2}\left[\left(\left|\eta_{1}\right|^{2}+\left|\eta_{2}\right|^{2}\right)^{2}+2\left|\eta_{1}\right|^{2}\left|\eta_{2}\right|^{2}\right],
\end{aligned}
$$

since at large $Q$ the oscillatory terms are wiped out when one integrates over $x$. In terms of $\eta_{a}$ 's the ground state of the resulting model is a ferromagnet. In other words, only one species of bosons condenses, e.g., $\eta_{1}$. The Hamiltonian for this field is just the bosonic one with a point-like repulsion. So the excitation spectrum at small wave vectors is linear in wave vector corresponding to the phase fluctuations of the condensate. Bosons of the second flavor remain massive with the energy gap $\Delta_{2} \sim\left|\eta_{1}\right|^{2} \sim \mu$.

Now we have to go back to the original variables. It turns out that the magnetic structure observed experimentally in Sul- $\mathrm{Cu}_{2} \mathrm{Cl}_{4}$ corresponds to the case denoted above as (i). For that case we get the following picture of the soft spin modes:

$$
\begin{gathered}
\mathbf{n}_{1}^{ \pm} \approx-\mathbf{l}_{1}^{ \pm} \approx \mathbf{N}_{1}^{ \pm}=\left(\eta_{1} \mathrm{e}^{\mathrm{i} Q x}, \eta_{1}^{*} \mathrm{e}^{-\mathrm{i} Q x}\right), \\
\mathbf{n}_{2}^{ \pm} \approx-\mathbf{l}_{2}^{ \pm} \approx \mathbf{N}_{2}^{ \pm}=\mathrm{i}\left(\eta_{1} \mathrm{e}^{\mathrm{i} Q x},-\eta_{1}^{*} \mathrm{e}^{-\mathrm{i} Q x}\right) .
\end{gathered}
$$

All spin fluctuations occur in the plane perpendicular to the magnetic field. Along the $J_{3}$ bonds the spins are antiparallel. Spins along $J_{2}$ or $J_{4}$ are perpendicular to each other. The propagation vector along the leg axis is $2 \pi l_{0}=\pi-Q$, so $l_{0}$ is close to $1 / 2$. Similarly, for (ii):

$$
\begin{aligned}
& n_{1}^{ \pm} \approx-n_{2}^{ \pm} \approx N_{1}^{ \pm}=\left(\eta_{1} \mathrm{e}^{\mathrm{i} Q x}, \eta_{1}^{*} \mathrm{e}^{-\mathrm{i} Q x}\right), \\
& l_{1}^{ \pm} \approx-l_{2}^{ \pm} \approx N_{2}^{ \pm}=\mathrm{i}\left(\eta_{1} \mathrm{e}^{\mathrm{i} Q x},-\eta_{1}^{*} \mathrm{e}^{-\mathrm{i} Q x}\right) .
\end{aligned}
$$

here the spins are parallel along $J_{2}$, and perpendicular on the $J_{3}$ bond.

The model thus qualitatively reproduces the diffraction result for the elastic modes, but also predicts that at $H>H_{c}$ the soft mode splits into two components. One, as required, is gapless. The other mode has a gap that scales as the square of the transverse ordered moment. Below we shall show that this prediction is indeed fully consistent with the inelastic data.

\section{DATA ANALYSIS}

A quantitative analysis of the inelastic neutron data involves fitting the measured scans to a parameterized model cross section function, numerically convoluted with the fourdimensional resolution function of the spectrometer. We start with the simpler regime $H<H_{c}$.

\section{A. Low fields}

For the vicinity of the minima of the one-dimensional dispersion where all scans were collected, the cross section was written in the single-mode approximation assuming three separate excitation branches corresponding to $S_{z}=0, \pm 1$. Since the Zeeman term commutes with the Heisenberg Hamiltonian, the spectrum at any $H<H_{c}$ will be exactly as at $H=0$, except for a constant shift in the energies of the $S_{z}=1$ and $S_{z}=-1$ magnons. With this in mind, the dispersion relations for mode $\alpha(\alpha=1,2,3)$ were written as:

$$
\hbar \omega_{\alpha, \mathbf{q}}=\hbar \omega_{\mathbf{q}}+\Delta_{\alpha}-\Delta_{0} .
$$

The spin wave velocity and zero-field gap were fixed at $v_{0}=14 \mathrm{meV}$ and $\Delta_{0}=0.52 \mathrm{meV}$, respectively, as previously measured in zero field. The gap energies $\Delta_{\alpha}$ for each mode were assumed to be field dependent. The single-mode cross section was then written as:

$$
\frac{d^{2} \sigma}{d \Omega d E^{\prime}} \propto \sum_{\alpha}|f(\mathbf{q})|^{2} \frac{A_{\alpha}}{\hbar \omega_{\mathbf{q}}} \delta\left(\omega-\omega_{\alpha, \mathbf{q}}\right) .
$$

Here are $A_{\alpha}$ are separate intensity prefactors for each of the three modes. This model, when convoluted with the known instrument resolution, fits all the scans collected at $H<H_{c}$ rather well. Such fits are shown in heavy solid lines in Fig. 2. Contributions due to the three components of the spectrum are represented by shaded areas. The asymmetric peak shapes with extended "tails" on the high-energy side are entirely due to resolution effects.

\section{B. $\boldsymbol{H}>H_{c}$ : low-energy modes}

Finding an appropriate dispersion relation for the two theoretically predicted descendants of the soft mode in the regime $H>H_{c}$ is not straightforward. The guidance provided by the calculations in the previous section is limited, as they are only applicable in the direct proximity of the ordering vector.

For the gapless mode it is clear that at $H=H_{c}$ one should still be able to employ Eqs. (1) and (15) with $\Delta_{1}=0$. However, for $H>H_{c}$ the dispersion near the ordering vector should be linear, with the slope progressively increasing with $H-H_{c}$. In the strongly $1 \mathrm{D}$ case like that of $\mathrm{Sul}-\mathrm{Cu}_{2} \mathrm{Cl}_{4}$, one can expect the dispersion relation to follow the lower bound of continuum excitations in isolated ladders. The latter were 
investigated in Ref. 13 and can be represented by the same parabolic curve as at $H=0$, but offset both in energy and momentum. The "gap" $\Delta_{1}$ becomes negative. The corresponding dispersion and neutron cross section are still given by Eqs. (15) and (16), respectively, but the definition of $\hbar \omega_{\mathbf{q}}$ in Eq. (15) is changed to:

$$
\begin{gathered}
\left(\hbar \omega_{\mathbf{q}}\right)^{2}=\Delta_{0}^{2}+v_{0}^{2}\left(\left|\mathbf{q} \mathbf{c}-2 \pi l_{0}\right|+\kappa\right)^{2}, \\
\kappa^{2}=\frac{\Delta_{0}\left|\Delta_{1}\right|}{v_{0}^{2}}\left(2-\frac{\Delta_{1}}{\Delta_{0}}\right) .
\end{gathered}
$$

Typical dispersion relations calculated using this equation are the lowest-energy curves plotted in the insets in Fig. 3.

We shall label the theoretically predicted gapped descendent of the soft mode with the index $\alpha=4$. It appears reasonable to approximate its dispersion with Eqs. (1) and (15), as at $H<0$. The gap $\Delta_{4}$ can be accurately determined from our scans that show a peak at the corresponding energy. In contrast, the parameter $\Delta_{1}$ actually represents the velocity at $\mathbf{q c} \rightarrow l_{0}$. It cannot be accurately measured due to limitations imposed by the experimental resolution. On the other hand, it cannot be totally disregarded either, as it affects the observable high-energy resolution tail of the gapless mode. In order to avoid dealing with an overparameterized model, a compromise was reached by somewhat arbitrarily postulating $\Delta_{4}=\left|\Delta_{1}\right|$. Indeed, both parameters are zero at $H=H_{c}$ and are expected to increase in absolute values at higher fields. As represented by the heavy solid lines in the lower-energy range of the plots shown in Fig. 3, this model, though somewhat artificial, reproduces the experimental data remarkably well. In the same figure the light gray shaded areas are partial contributions of the gapless and gapped components (modes 1 and 4), respectively.

\section{C. $\boldsymbol{H}>\boldsymbol{H}_{c}$ : high energies}

Upon crossing the critical field, the central component of the excitation triplet undergoes some drastic changes. This mode is the strongest and sharpest feature of the spectrum at $H<H_{c}$ [Fig. 2(c)], but is replaced by a much broader peak above the transition [Fig. 3(a)], measured using in the same experimental configuration). The single-mode approximation (16) gives excellent fits to the data at low fields, but totally fails to describe the shape of the middle mode at $H>H_{c}$ (Fig. 3, dashed curves). This leads us to the conclusion that the quasiparticle description of this part of the spectrum breaks down in the high-field phase. Instead, the excitations are a broad continuum of states. By analogy with the Müller ansatz that describes continuum excitations in gapless spin chains, we used the following empirical expression to approximate the corresponding contribution $(\alpha=2)$ of this spectral feature to the neutron cross section:

$$
\frac{d^{2} \sigma}{d \Omega d E^{\prime}} \propto A_{2}|f(\mathbf{q})|^{2}\left(\omega-\omega_{2, \mathbf{q}}\right)^{-\zeta} \Theta\left(\omega-\omega_{2, \mathbf{q}}\right) .
$$

In this formula $\hbar \omega_{2, \mathbf{q}}$ is still given by Eq. (15), and describes the lower bound of the continuum. A parameter $\zeta$ characterizes how steeply the intensity falls off with energy. With the

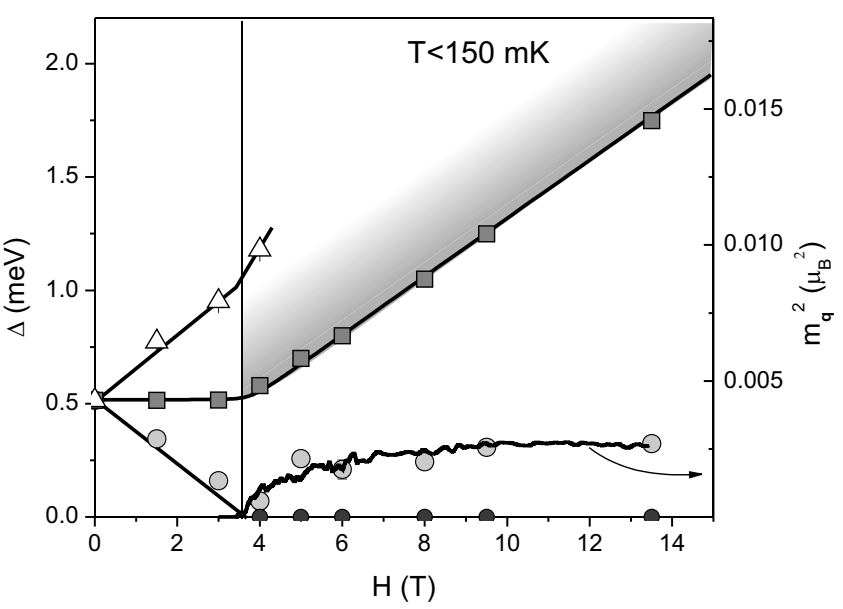

FIG. 4. Field dependence of the excitation energies at the 1D AF zone center in $\mathrm{Sul}-\mathrm{Cu}_{2} \mathrm{Cl}_{4}$. The points are the data from the INS experiments and the solid lines are guides for the eye. At $H>H_{c}$, the central excitation branch is replaced by a sharp continuum represented by the shaded band. The field dependence of the gapped excitation (above $3.7 \mathrm{~T}$ ) is compared with that of the square of the transverse ordered moment, from Ref. 9.

value $\zeta=0.30(6)$ refined at $H=9.5 \mathrm{~T}$, this formula yields much better fits to the data at all fields at $H>H_{c}$ (Fig. 3, solid curves) than the single-mode approximation (dashed curves).

The highest-energy component of the original triplet of gap excitations is also strongly affected by the transition. As clearly seen in Fig. 3, its intensity drops dramatically and it is no longer observed at $H>4 \mathrm{~T}$. To describe the mode at that field however, in our analysis we employed an expression similar to that for the middle mode, the parameter $\zeta$ fixed at the same value.

\section{Fits to experimental data}

The model cross sections described above were numerically convoluted with the spectrometer resolution function, calculated in the Popovici approximation. ${ }^{14}$ They were then used in the least-squares analysis of constant- $Q$ scans collected at each value of the applied field. The resulting fits are shown as the solid lines in Figs. 2 and 3. Partial contributions of each spectral component are represented by the shaded areas. The insets show the dispersion relations of all components calculated using the parameters determined in the fitting process. The shaded area signifies continuum scattering, with the corresponding solid line indicating the lower bound. In the same plot we show the evolution of the experimental resolution ellipse in the course of each constant- $Q$ scan. The gap energies obtained in these fits are plotted vs. applied magnetic field on Fig. 4 (symbols).

\section{DISCUSSION}

Despite fact that our data analysis is based on several somewhat arbitrary assumptions regarding the form of the dynamic structure factor, the extracted field dependencies of the gap energies should be quite reliable. Indeed, the gaps are 
robustly identified by a sharp jump in the neutron intensity that occurs in the energy window defined by the resolution function. The fact that the central excitation branch is replaced by a sharp continuum at $H>H_{c}$ is also a solid experimental finding, as is the disappearance of the higher-energy mode.

The linear Zeeman splitting of the triplet at $H<H_{c}$ occurs exactly as expected: the system is not ordered and the Zeeman term commutes with the Heisenberg Hamiltonian. For $H>H_{c}$, the central prediction of our Ginzburg-Landau analysis is that the energy of the gapped excitation scales as the square of the transverse ordered moment. This is indeed consistent with the experiment. In Fig. 4 the heavy solid line, and the corresponding right-hand-side $Y$ axis, represent the field dependence of the latter, as previously measured experimentally. ${ }^{9}$ To within a constant scaling factor, this curve exactly reproduces the field dependence of the reopening gap $\Delta_{4}(H)$ measured in this work.

The dramatic broadening of the higher-energy excitations at $H>H_{c}$ is not captured by our hydrodynamic model. While a further theoretical study is clearly required, we note that the phenomenon resembles the one previously observed in the bond-alternating $S=1$ chain system NTENP. ${ }^{15}$ In the lat- ter system it was attributed to magnon decay channels that are opened by the lifting of rotational symmetry at $H_{c}$. A similar mechanism may at work in $\mathrm{Sul}-\mathrm{Cu}_{2} \mathrm{Cl}_{4}$.

\section{CONCLUSION}

In summary, we suggest that the geometric frustration and the coupled-ladder topology of $\mathrm{Sul}-\mathrm{Cu}_{2} \mathrm{Cl}_{4}$ are responsible for a complex and very unusual excitation spectrum in the magnetized state. At the same time, triplet low-energy excitations at $H<H_{c}$ are rather typical for an isotropic spin liquid.

\section{ACKNOWLEDGMENTS}

The work at Oak Ridge National Laboratory was sponsored by the Scientific User Facilities Division, Office of Basic Energy Sciences, U. S. Department of Energy. U.S. DOE ORNL is operated by UT-Battelle, LLC for the U.S. DOE under Contract No. DE-AC05-00OR22725. A.M.T. acknowledges the support from U.S. DOE under contract number DE-AC02-98 CH 10886.
*Present address: Laboratory for Neutron Scattering, ETH Zürich \& Paul Scherrer Institut, Villigen, Switzerland.

${ }^{1}$ T. Giamarchi and A. M. Tsvelik, Phys. Rev. B 59, 11398 (1999).

${ }^{2}$ M. Rice, Science 298, 760 (2002).

${ }^{3}$ T. Giamarchi, C. Ruegg, and O. Tchernyshyov, Nat. Phys. 4, 198 (2008).

${ }^{4}$ M. Matsumoto, B. Normand, T. M. Rice, and M. Sigrist, Phys. Rev. Lett. 89, 077203 (2002).

${ }^{5}$ V. O. Garlea, A. Zheludev, T. Masuda, H. Manaka, L.-P. Regnault, E. Ressouche, B. Grenier, J.-H. Chung, Y. Qiu, K. Habicht, K. Kiefer, and M. Boehm, Phys. Rev. Lett. 98, 167202 (2007).

${ }^{6}$ A. Zheludev, V. O. Garlea, T. Masuda, H. Manaka, L.-P. Regnault, E. Ressouche, B. Grenier, J.-H. Chung, Y. Qiu, K. Habicht, K. Kiefer, and M. Boehm, Phys. Rev. B 76, 054450 (2007).

${ }^{7}$ M. Fujisawa, J.-I. Yamaura, H. Tanaka, H. Kageyama, Y. Narumi, and K. Kindo, J. Phys. Soc. Jpn. 72, 694 (2003).
${ }^{8}$ V. O. Garlea, A. Zheludev, L.-P. Regnault, J.-H. Chung, Y. Qiu, M. Boehm, K. Habicht, and M. Meissner, Phys. Rev. Lett. 100, 037206 (2008).

${ }^{9}$ V. O. Garlea, A. Zheludev, K. Habicht, M. Meissner, B. Grenier, L.-P. Regnault, and E. Ressouche, Phys. Rev. B 79, 060404(R) (2009).

${ }^{10}$ D. G. Shelton, A. A. Nersesyan, and A. M. Tsvelik, Phys. Rev. B 53, 8521 (1996).

${ }^{11}$ I. Affleck, Phys. Rev. B 41, 6697 (1990).

${ }^{12}$ A. A. Nersesyan, A. O. Gogolin, and F. H. L. Essler, Phys. Rev. Lett. 81, 910 (1998).

${ }^{13}$ O. P. Sushkov and V. N. Kotov, Phys. Rev. Lett. 81, 1941 (1998).

${ }^{14}$ M. Popovici, Acta Crystallogr., Sect. A: Cryst. Phys., Diffr., Theor. Gen. Crystallogr. 31, 507 (1975).

${ }^{15}$ L.-P. Regnault, A. Zheludev, M. Hagiwara, and A. Stunault, Phys. Rev. B 73, 174431 (2006). 